\title{
EL EFECTO DE LA MATERNIDAD SOBRE LOS SALARIOS FEMENINOS EN ECUADOR ${ }^{1}$
}

\author{
Por: Héctor Alberto Botello² - Andrea López Alba ${ }^{3}$
}

\section{RESUMEN}

En el presente trabajo se analiza el efecto de la maternidad sobre los salarios femeninos del año 2012 en el Ecuador estudiando sobre la tenencia, el número y la estructura de edad de los hijos. La metodología implementa ecuaciones mincerianas sobre los datos del ingreso laboral provenientes de las encuestas de hogares; para posteriormente descomponer el diferencial con el método de Oaxaca Blinder con corrección sobre el sesgo de selección. Los resultados muestran que las madres ganan en promedio 7,56\% menos que las no madres, presentándose una alta heterogeneidad entre las provincias analizadas y los niveles educativos. Luego de controlar la edad de los hijos se aprecia que las madres de niños de menos de 5 años ganan un $10 \%$ menos, mientras que las madres que tienen niños de más de 5 años, la disminución es de solo el $2 \%$. Por número, si la mujer tiene a su cuidado dos hijos la brecha por maternidad promedio es del 3,7\% y si tiene más de dos hijos la rebaja es del 2,26\%. No obstante, la mayor parte de la brecha por maternidad en el Ecuador no está causada por el hecho de ser madres sino por sus características socioeconómicas intrínsecas.

Palabras claves: Maternidad, brecha salarial, Ecuación Minceriana, Descomposición Oaxaca Blinder, Ecuador, discriminación laboral.

Clasificación JEL: J13, J16, J24, C24

1. El presente artículo hace parte del trabajo de investigación sobre el desarrollo económico en el Ecuador impulsado y promovido por la Universidad Industrial de Santander. Iniciado en el 2012.

2. Profesor de Política Monetaria y Fiscal. Universidad Industrial de Santander. Estudiante de Maestría Ingeniería Industrial. Universidad Industrial de Santander. Universidad Industrial de Santander. hectoralbertobotello@gmail.com

3. Estudiante de Economía Universidad Industrial de Santander. andrealopezalba@gmail.com Artículo recibido: 8 de enero de 2015. Aprobación definitiva: 11 de marzo de 2015. 


\title{
THE EFFECT OF MOTHERHOOD ON WAGES IN ECUADOR
}

\author{
By: Héctor Alberto Botello - Andrea López Alba
}

\begin{abstract}
This paper analyses the effect of motherhood on women's wages in 2012 in Ecuador studying tenure, the number and age structure of the children is analyzed. The methodology implemented Mincerian equations on labor income data from household surveys; to further decompose the differential method Oaxaca Blinder corrected for selection bias. The results show that mothers earn on average $7.56 \%$ less than non-mothers, presenting a high heterogeneity among provinces analyzed and educational levels. After controlling for age of the children shown that mothers of children under five years earn $10 \%$ less, while mothers with children over five years, the decrease is only $2 \%$. By number if the woman has two children in their care gap for maternity average is $3.7 \%$ and if you have more than two children rebate is $2.26 \%$. However, most of the gap maternity in Ecuador is not caused by the fact that mothers but for its intrinsic socioeconomic characteristics.
\end{abstract}

Keywords: Motherhood, wages gap, Mincerian equation, Oaxaca Blinder decomposition, Ecuador, employment discrimination.

JEL Classification: J13, J16, J24, C24 


\section{Introducción}

La literatura coincide en que existe un diferencial salarial negativo hacia las mujeres en el mercado laboral asociada a la discriminación de género (Atucha, 2009; Martínez y Acevedo, 2004; Fuentes, Palma y Montero, 2005; Peñas, 2002; Rivera, 2013). Esta situación es de interés social ya que según la CEPAL, en el 2012, en el $30 \%$ de los hogares de América Latina el jefe de hogar era mujer y era la principal aportante de los ingresos en el mismo.

En la literatura una de las explicaciones de esta brecha es la maternidad (Budig y England, 2001; Molina y Montuenga, 2008). La crianza de los hijos recae especialmente en las mujeres, por lo que se sufren costes de oportunidad al realizar esta actividad en términos de tiempo y esfuerzo, afectando negativamente los ingresos laborales. Por ende, las mujeres que tienen hijos y participan en el mercado laboral tienden a ser menos productivas en el trabajo ya que se encuentran en empleos que permiten horarios flexibles y retribuyen bajas remuneraciones que les son consecuentes (Olarte y Peña, 2010). Igualmente, estas mujeres no pueden aceptar empleos donde se presenten altas responsabilidades y que ocupen mucho tiempo ya que deben dedicar su atención simultáneamente al trabajo y sus hijos.

En este orden de ideas, el análisis de estas diferencias resulta relevante en la formulación de políticas públicas enfocadas hacia la equidad de género y el desarrollo incluyente. Dado que el menor ingreso de las madres, eleva la probabilidad de que los hogares donde son las principales perceptoras del ingreso, continúen en la pobreza.

En términos empíricos, los estudios iniciales de Mincer (1974) y Becker (1985) explicaban que la brecha salarial por maternidad se debía a las diferencias en la experiencia laboral de madres y no madres, ya que las primeras dedicaban la mayor parte de su tiempo a la crianza de sus hijos. No obstante, literatura reciente (Jacobsen y Levin, 1995; Korenman y Neumark, 1992; Waldfogel, 1995; Baxter, 1992; Joshi y Newell, 1989) encuentra que aún eliminando el efecto de la experiencia de la estimación, la brecha prevalece. Tal es el caso de Estados Unidos donde Budig y England (2001) utilizando la encuesta longitudinal nacional de la juventud desde 1982 a 1993 e implementando un modelo de efectos fijos, encuentran que la penalización salarial es del 7\% por hijo y con el control de la experiencia laboral, esta se reduce a 5\%. Todd (2001) realizó un análisis semejante en Reino Unido y Australia donde las madres que poseen dos hijos tienen mayores pérdidas en salario de alrededor del 25,5\% y 12\%, respectivamente; posteriormente en Estados Unidos y Alemania calculó que las diferencias se ubicaban en un 10.5\% y 10.7\% en cuanto la pena salarial por hijo y controlando por experiencia laboral, correspondientemente. Por su parte, Harkness y Waldfogel (2003) calculan con base en microdatos de la encuesta de empleo y salarios, que los países que presentan menos diferencias salariales son Canadá, Finlandia y Suecia, inferiores al 5\%. En el caso de España, Molina y Montuenga (2008) encuentra que incluyendo variables observables y no observables, las madres pierden el 9\% de su salario normal. Este valor incrementa a medida que se tienen más hijos.

Para América Latina, Piras y Ripani (2005) estiman la brecha por maternidad para Bolivia, Brasil, Ecuador y Perú con los datos de la Encuesta de Hogares de 
cada país de 1999. El modelo estimado usa una función estándar de ingresos con base en los trabajos de Mincer (1974). Los resultados sugieren que en Perú existe una penalización salarial de 11\% aproximadamente por la presencia de un hijo, mientras que en Brasil, por el contrario, existe una ganancia de $8,1 \%$ por la presencia de dos o más hijos. En Ecuador y Bolivia, la brecha por maternidad no fue estadísticamente significativa; concluyen entonces que los resultados para Latinoamérica no manifiestan un claro impacto de la maternidad sobre los salarios a diferencia de las muestras en los países desarrollados.

En países latinoamericanos, existen pocos trabajos específicos, como el de Olarte y Peña (2010) en Colombia, donde evidenciaron que las madres ganan en promedio $17.6 \%$ menos que las no madres. Luego de controlar por variables observables, aún persiste una brecha salarial de $9.4 \%$ entre madres y no madres; ésta se hace más alta (18.4\%) cuando los hijos tienen menos de 5 años de edad. Es importante subrayar que el efecto de estos resultados es más notorio en madres con hijos entre 0 y 5 años.

De esta revisión se observa que es necesario ampliar y actualizar los trabajos a nivel regional para cuantificar el impacto que tiene la brecha salarial por maternidad. Por tanto, en el presente trabajo se analizó el efecto de la maternidad sobre los salarios en el Ecuador, estudiando sobre la tenencia del número de hijos y de la estructura de las edades de los mismos en el 2012, para lo cual, las secciones están organizadas de la siguiente manera. En la introducción además se describen las principales contribuciones al tema halladas en la literatura internacional. La sección 2 detalla la metodología utilizada y se hace una descripción de los datos. Posteriormente, en la sección 3 se presentan los resultados. Finalmente en la sección 4 y 5 se realizan las conclusiones y la bibliografía, respectivamente.

\section{Metodología}

\subsection{Modelo de Mincer}

El modelo de Mincer (1974), involucra las características de los trabajadores como determinantes de los ingresos laborales de los ocupados, es uno de los modelos más explorados y conocidos en la literatura sobre economía laboral. (Psacharopoulos, 2004; Cohn y Addison, 1998; Asplund y Pereira, 1999; Harmon et al, 2001, 2003).

Este modelo parte de una función semi-logarítmica (ecuación 1) que se estima por medio del procedimiento de mínimos cuadrados ordinarios (MCO), teniendo como variable dependiente el logaritmo de los salarios de los trabajadores y como variables independientes los años de educación del trabajador, la experiencia laboral (y su cuadrado), la rama de actividad, su ocupación, el lugar de trabajo, el tamaño de la empresa, la tenencia de contrato, entre otros.

$$
\log (Y)=\beta 0+\beta 1 S+\beta 2 \operatorname{Exp}+\beta 3 \operatorname{Exp} 2+\beta 4 L+\varepsilon(1)
$$

- $Y$ es el salario del individuo

- $S$ es el número de años de educación formal completada 
- Exp son los años de experiencia laboral

- L corresponde a otras variables del trabajador.

- $\varepsilon$ es el término de perturbación aleatoria que se distribuye según una Normal

Entre las otras variables del trabajador que se van a controlar en este estudio están la provincia donde habita el individuo, estado civil, etnia, tamaño de la empresa donde labora, realización de oficios en el hogar, rama de actividad, satisfacción laboral, obtención de subsidios del gobierno.

Se pueden diseñar diferentes especificaciones de la ecuación de Mincer en el término de $L$ para analizar las distintos tipos de brechas de maternidad en pro de controlar la edad y la cantidad de niños al cuidado de la mujer, ya que estas características influyen sobre el tiempo y la atención que las madres deben poner en sus hogares. Este artículo emplea cuatro especificaciones: en la forma inicial se valora únicamente si la madre tiene hijos o no con una variable dicotómica, que es igual a cero si la mujer no tiene hijos y uno en caso contrario. En la segunda se crea una variable categórica que tiene el valor de cero si la mujer no tiene hijos, uno si tiene hijos de menos de 5 años y toma el valor de dos si la mujer tiene niños de más de cinco años. En la tercera se introduce una variable escalar continua que mide el número de niños dentro del hogar. Finalmente, en la cuarta especificación se calcula el efecto de tener uno, dos o más de dos hijos.

En términos de interpretación los coeficientes $(\beta)$ representan la variación porcentual sobre el ingreso laboral que tendrá en cada uno de los cambios en las características de los trabajadores. La efectividad de este modelo se mide a través de la varianza de la variable dependiente captada por parte de las variables de control. Este indicador se denomina $\mathrm{R}^{2}$. Si este es más alto, más efectivo es el modelo en determinar el comportamiento de la variable dependiente. Hay diferentes situaciones sobre la naturaleza de los datos que también se deben arreglar para una correcta calibración del modelo tales como la presencia de heterocedasticidad, multicolinealidad y autocorrelación serial.

Asimismo, es fundamental corregir un problema adicional que se encuentra implícito en los datos, el denominado sesgo de selección. Lo que consiste en la ausencia de aleatoriedad muestral, o selección muestral no aleatoria dentro de las encuestas de hogares que se realizan (Esquivel, 2007; Rivera, 2013). Se denomina sesgo, porque una muestra que esté concentrada en cierto grupo poblacional desvía el resultado obtenido de una variable de interés hacia el grupo que tenga mayor representación. Este problema es necesario de corregir cuando se investiga sobre diferencias en los ingresos laborales de las personas sobre grupos de población específicos, ya que no permite obtener resultados exactos del fenómeno analizado. La corrección de Heckman es la solución más utilizada en la literatura para efectos de estimación (Heckman, 1979; Rivera, 2013), método que se realiza estimando dos regresiones: la primera estimación se realiza a base de la ecuación de participación, entendida como la probabilidad de que el individuo participe en el mercado laboral contra los factores que pueden incidir en la elección.

$$
\mathrm{pi}=\beta \mathrm{o}+\mathrm{zi} \varphi+\mathrm{ui}
$$


Donde pi se refiere a la probabilidad de participar en el mercado laboral, zi corresponde a un vector con variables explicativas que influyen en la decisión de participar, $\varphi$ es un vector de parámetros, y ui son los errores. Después de realizada, el segundo cálculo es la ecuación de Mincer incorporando la corrección de selección realizada por lambda ( $\lambda$ ) como inversa del ratio de Mill (Rivera, 2013):

$$
\log (\mathrm{Y})=\beta 0+\beta 1 S+\beta 2 \operatorname{Exp}+\beta 3 \operatorname{Exp} 2+\beta 4 \mathrm{~L}+\lambda \operatorname{ti} \theta+\varepsilon i(3)
$$

Con ello se corrige el sesgo de selección. Usando el argumento de Torres y Celton (2009), la importancia del uso de $\lambda$ se determina al estimar la regresión, siempre y cuando su coeficiente sea significativo. En el caso de no ser representativo, se podría trabajar directamente sin la necesidad de la corrección.

\subsection{Modelo Oaxaca Blinder}

A partir de la estimación del diferencial con la ecuación de Mincer se puede observar el origen del mismo, es decir, si la brecha salarial entre las madres y no madres dependen de las características de las mujeres (variables observables) o de la condición de control (ser madre, variable no observable). Para averiguar lo anterior se utiliza la metodología de descomposición salarial de Oaxaca- Blinder.

Resumiendo la demostración, se toma la explicación de Espinoza (2009) y de Rivera (2013) que empleándola en nuestro caso de estudio, las ecuaciones de salario, con las mismas variables de la ecuación anterior para madres y no madres, se espera encontrar la diferencia dentro del valor esperados de los salarios.

$$
Y_{i}=X_{i} \beta_{i}+u_{i} / E\left(u_{i}=0\right) / i \in[A, B]
$$

Siendo A y B los grupos a comparar. La descomposición de Oaxaca-Blinder consiste en la siguiente operación:

$$
R=E\left(Y_{A}\right)-E\left(Y_{B}\right)
$$

Si se reemplaza 1 en 2 y se asume $E\left(u_{i}=0\right)$, se tiene que:

$$
R=E\left(X_{A}\right)^{\prime} \beta_{A}-E\left(X_{B}\right)^{\prime} \beta_{B}(6)
$$

Para entender todos los efectos que se pueden extraer de la ecuación anterior, se puede realizar la descomposición algebraica expuesta por Daymont y Andrisani $(1984)^{4}$ :

$$
R=\left[E\left(X_{A}\right)-E\left(X_{B}\right)\right]^{\prime} \beta_{A}+\left[E\left(X_{A}\right)^{\prime}\left(\beta_{A}-\beta_{B}\right)\right]+\left[E\left(X_{A}\right)-E\left(X_{B}\right)\right]^{\prime}\left(\beta_{A}-\beta_{B}\right)
$$

4. Para mayor ampliación de la explicación matemática, se puede revisar el estudio de Daymont y Andrisani (1984), en el pie de página 6 del documento. 
En la ecuación anterior, el primer término de la derecha corresponde a diferencias en las características entre los dos grupos, el segundo término se refiere a diferencias en los rendimientos de esas características, y el tercer elemento es la interacción causada por una diferencia simultánea en las características y retornos. En este punto hay que mencionar que existe otro tipo de descomposición la cual parte de la existencia de un conjunto de coeficientes que no son causantes de las brechas salariales por maternidad, pero al mismo tiempo son claves para encontrar las diferencias en los predictores. Supóngase que $\beta^{*}$ es un coeficiente hipotético que se puede reemplazar en la ecuación (7) como sigue:

$$
R=\left[E\left(X_{A}\right)-E\left(X_{B}\right)\right]^{\prime} \beta^{*}+\left[E\left(X_{A}\right)^{\prime}\left(\beta_{A}-\beta^{*}\right)\right]+\left[E\left(X_{A}\right)-E\left(X_{B}\right)\right]^{\prime}\left(\beta_{A}-\beta^{*}\right)
$$

A partir de la ecuación (8) se pueden extraer dos efectos, la primera parte es la parte de la desigualdad que es explicada por las diferencias en los predictores. El segundo efecto es la unión de los dos últimos términos:

$$
U=\left[E\left(X_{A}\right)^{\prime}\left(\beta_{A}-\beta^{*}\right)\right]+\left[E\left(X_{A}\right)-E\left(X_{B}\right)\right]^{\prime}\left(\beta_{A}-\beta^{*}\right)
$$

La ecuación 9 se refiere a la parte no explicada por los predictores, la cual se le atribuye, frecuentemente, a la tenencia de hijos. No obstante ésta también captura el efecto de variables no especificadas dentro del modelo. Montenegro (2001) menciona que el segundo coeficiente es la parte del diferencial que no se explica por las variables contenidas dentro del modelo y se debe a factores no observables, asociados a la maternidad. Adicionalmente a esta metodología se le suele aplicar el sesgo de corrección respectivo.

\subsection{Fuentes de datos}

Los datos utilizados en el presente estudio se obtuvieron en términos trimestrales de la Encuesta de Empleo, desarrolladas por el Instituto Ecuatoriano de Estadísticas (INEC) del año 2012. La población total de la encuesta es de 191 mil personas con un rango total ajustado con factores de expansión a un poco más de 48 millones de personas. Con estos datos se puede separar a la población objeto del análisis, mostrándose los resultados en la tabla 1.

Tabla 1. Distribución de los encuestados por país

\begin{tabular}{lrrr}
\hline \multicolumn{1}{c}{ Agregado } & Hombre & \multicolumn{1}{c}{ Mujeres } & \multicolumn{1}{c}{ Total } \\
\hline Total de personas & $23,805,480$ & $24,843,441$ & $48,648,921$ \\
\hline PET & $19,863,283$ & $21,026,912$ & $40,890,194$ \\
\hline PEA & $13,641,874$ & $9,288,066$ & $22,929,940$ \\
\hline Desempleados & 524,511 & 474,419 & 998,930 \\
\hline Ocupados & $13,117,363$ & $8,813,647$ & $21,931,010$ \\
\hline Tienen Hijos (\% PEA) & $47.56 \%$ & $46.45 \%$ & $47.10 \%$ \\
\hline
\end{tabular}

Fuente: Encuestas de Empleo Ecuador 2012. INEC 
De estos datos, se manifiesta que 8.8 millones de mujeres están ocupadas en el mercado laboral, siendo el 46,5\% madres. Asimismo, se puede estimar que la tasa de ocupación laboral femenina es de aproximadamente 42\%. A continuación se hace un análisis descriptivo más detallado de este grupo poblacional.

\subsection{Análisis descriptivo de los datos}

En la tabla 2 se muestran las estadísticas descriptivas de la base de datos de los hogares ecuatorianos con mujeres ocupadas que tienen hijos contra los que no tienen hijos. Las no madres tienen una media de edad 10 años más alta aunque tienen una desviación estándar cercana a los 15 años. Mientras que la media de la edad para las madres es de 44 años con una desviación de 10 años. Asimismo se observa un alto grado de madres solteras trabajadoras, ya que cerca del $69 \%$ de las madres encuestadas no viven con su pareja.

Tabla 2. Análisis descriptivo de los hogares de las mujeres que tienen y no tienen hijos y que participan en el mercado laboral

\begin{tabular}{|c|c|c|c|}
\hline Característica & Característica & No Tienen & Tienen \\
\hline Edad & Media & 54.08 & 44.64 \\
\hline \multicolumn{2}{|r|}{ Desviación } & 15.53 & 11.67 \\
\hline Vive con la pareja & $\mathrm{Si}$ & $60.83 \%$ & $69.86 \%$ \\
\hline Máximo Nivel Educativo & Ninguno & $11.71 \%$ & $4.26 \%$ \\
\hline \multirow{7}{*}{ Alcanzados } & Primaria & $37.74 \%$ & $33.94 \%$ \\
\hline & Educación Básica & $0.71 \%$ & $0.66 \%$ \\
\hline & Secundaria & $25.62 \%$ & $35.31 \%$ \\
\hline & Educación Media & $0.59 \%$ & $0.89 \%$ \\
\hline & Superior no universitario & $0.88 \%$ & $1.05 \%$ \\
\hline & Superior Universitario & $19.62 \%$ & $21.78 \%$ \\
\hline & Post-grado & $1.87 \%$ & $1.36 \%$ \\
\hline \multirow[t]{4}{*}{ Tipo de Trabajador } & Cuenta propia & 43.58 & 40.12 \\
\hline & Patrona o Empleadora & 3.59 & 3.00 \\
\hline & Obrera o Empleada & 30.36 & 36.10 \\
\hline & Hogar No remunerado & 22.47 & 20.78 \\
\hline \multirow[t]{2}{*}{ Jornada Laboral } & Completa & 63.10 & 59.31 \\
\hline & Parcial & 36.90 & 36.90 \\
\hline Tenencia Seguridad Social & $\mathrm{Si}$ & 54.71 & 52.30 \\
\hline
\end{tabular}


Revista TENDENCIAS Vol. XVI No. 1

\begin{tabular}{|c|c|c|c|}
\hline \multirow[t]{8}{*}{ Tamaño de la empresa } & Una persona & 43.51 & 38.70 \\
\hline & De 2 a 3 personas & 27.22 & 25.34 \\
\hline & De 4 a 5 personas & 4.89 & 6.67 \\
\hline & De 6 a 10 personas & 3.02 & 4.38 \\
\hline & De 11 a 20 personas & 2.35 & 3.19 \\
\hline & De 21 a 50 personas & 2.33 & 3.02 \\
\hline & De 51 a 100 personas & 0.76 & 0.91 \\
\hline & Más de 100 personas & 15.92 & 17.79 \\
\hline \multirow[t]{3}{*}{ Lugar de trabajo } & Vivienda Propia o Ajena & 49.14 & 42.64 \\
\hline & Local de la empresa & 43.48 & 51.20 \\
\hline & Ambulante & 7.38 & 6.16 \\
\hline \multirow[t]{8}{*}{ Rangos Ingreso Laboral } & Menos de $\$ 200$ & $39.56 \%$ & $42.61 \%$ \\
\hline & $\$ 201-\$ 400$ & $29.46 \%$ & $32.08 \%$ \\
\hline & $\$ 401-\$ 600$ & $11.62 \%$ & $11.01 \%$ \\
\hline & $\$ 601-\$ 800$ & $6.33 \%$ & $5.52 \%$ \\
\hline & $\$ 801-\$ 1000$ & $4.83 \%$ & $3.67 \%$ \\
\hline & $\$ 1001-\$ 1200$ & $2.43 \%$ & $1.67 \%$ \\
\hline & $\$ 1201-\$ 1400$ & $1.52 \%$ & $0.91 \%$ \\
\hline & Más de $\$ 1401$ & $4.24 \%$ & $2.53 \%$ \\
\hline
\end{tabular}

Fuente: Encuesta de empleo Ecuador 2012. INEC.

En relación a la educación se estima que las mujeres que tienen hijos poseen mayores niveles de educación; un 21\% de estas cursaron grado universitario contra el 19\% de las que no tienen hijos. Igualmente, mientras que el 12\% de las no madres, no tienen ningún nivel educativo, solo un $4 \%$ de las madres padecen de esta situación.

En resumen, en el análisis socioeconómico se observa que las madres ocupadas poseen mejores niveles de educación, en comparación con las que no son madres, y a primera vista esta característica se ve reflejada en sus ingresos. No obstante es necesaria una revisión de las características laborales para especificar otras fuentes de esta afirmación.

A continuación se describen las diferentes características laborales de las madres y no madres para complementar la anterior idea. 


\subsection{Análisis de Estadísticas laborales}

Las madres de las ciudades ecuatorianas encuestadas suelen ser relativamente menos independientes que su contraparte (no madres). Por ejemplo, el $40 \%$ de las madres son trabajadoras por cuenta propia contra el $44 \%$ de las no madres. Mientras que un 3\% son patronas contra el 3,6\% de las que no son madres. En este sentido, los grupos comparados operan sobre una jornada laboral similar ya que el 59\% de las mujeres que tienen hijos y el 63\% de las que no tienen, laboran en jornada completa.

Sin embargo, según estas ocupaciones no poseen los mismos niveles de seguridad social. Solo el $52 \%$ de las madres poseen este beneficio mientras que el 54\% de las no madres lo poseen.

Para más del 75\% de las mujeres ocupadas que tienen hijos sus labores se desempeñan en empresas de menos de 10 personas contra el $78 \%$ de las que no tienen hijos. En las empresas de más de 51 personas, solo laboran un 16\% de las no madres contra un 19\% de las madres. En términos de ubicación, un 35\% de las madres labora en empresas informales dentro de la vivienda propia o ajena frente al 19,7\% de las no madres. El 13,5\% de las madres tienen puestos ambulantes y un $52 \%$ laboran en locales fijos de las empresas contra el $72 \%$ de las mujeres que no tienen hijos.

De este análisis descriptivo se puede determinar que en Ecuador en general las madres trabajadoras que laboran poseen características muy similares a las que no tienen hijos. Esta situación es muy diferente a la encontrada en otros ejercicios (Olarte y Peña 2010; Waldfogel 1995, 1997; Baum, 2003; Lai y Masters, 2005). Esto indica que las madres poseen mejores pero leves diferencias frente a las no madres. Están localizadas en la parte formal de la economía, no obstante cuando se observan en los rangos de los ingresos laborales en dólares de 2012 se aprecia que ganan en promedio menores salarios. Por ejemplo, según la encuesta del INEC, en 2012 el 75\% de las madres ganan menos de 400 dólares al mes contra el 69\% de las mujeres no madres. Esta situación existe a pesar de la mayor dotación de las madres en el mercado laboral lo que parece indicar que existe una leve presencia de discriminación en el mercado laboral ecuatoriano hacia las madres. Sin embargo es necesario la aplicación de modelos que cuantifiquen correctamente la brecha de salarios por maternidad en el ecuador en el 2012.

\section{Resultados y análisis}

\subsection{Modelo de Mincer}

En la Tabla 3 se muestran los resultados de las estimaciones sobre la ecuación 1 con cada una de las especificaciones enunciadas en la sección anterior, usando las variables seleccionadas mediante los datos disponibles para las mujeres ocupadas dentro de la encuesta de trabajo del INEC (Ecuador) para el año 2012. Se exponen las observaciones totales, el $\mathrm{R}^{2}$ ajustado y las variables tanto continúas como categóricas, sus características y el efecto porcentual que tienen sobre el ingreso laboral promedio. 
Después de depurar la base de datos y extraer las observaciones perdidas, dispersas, se analizaron los salarios de cerca de 3,4 millones de mujeres ecuatorianas trabajadoras. El porcentaje de varianza estimado por el modelo de Mincer es cercano al $80 \%$, siendo el mejor modelo la especificación 2 referida al análisis de tenencia de hijos por edad. Este porcentaje de varianza explicada fue significativamente mayor, al ser comparado con otros trabajos de la literatura (Budig y England, 2001; Harkness y Waldfogel, 2003; Korenman, y Neumark, 1992; Molina y Montuenga, 2008). Esto se debe principalmente a la introducción de suficientes variables explicativas del comportamiento del salario de las mujeres ocupadas.

En relación a su interpretación, los coeficientes de las variables continuas reflejan cómo afecta porcentualmente un cambio de $1 \%$ de éstas respecto a la variable independiente. Por ejemplo, un aumento del $1 \%$ en las horas de trabajo, incrementan en un $0,0137 \%$ el ingreso laboral promedio. Por otro lado, en las variables categóricas el cambio porcentual sobre el puntaje se da con relación a una característica base. Por ejemplo, la tenencia de un grado educativo de postgrado incrementa entre un 50\% el ingreso laboral, en comparación con las que no han cursado ningún grado escolar.

Sobre las variables de interés para la hipótesis del estudio, la tenencia de hijos es origen de una brecha salarial cercana al 2\% (especificación 1). Sin embargo, cuando se interactúa este efecto con la educación de la madre se observa que la brecha por maternidad no se distribuye homogéneamente. Este fenómeno parece afectar significativamente a las mujeres con grados universitarios, con sus salarios un $11 \%$ por debajo de las no madres. Mientras que en los extremos de la distribución, ningún grado educativo y estudios de postgrado, las madres poseen ingresos entre un $16 \%$ y $19 \%$ por encima de las mujeres que no tienen hijos. Asimismo, cuando se realiza una exploración geográfica del diferencial de las madres (gráfica 3), se encuentran regiones que tienen un mayor diferencial en contra de las madres, tales como las provincias localizadas al nororiente del país Manabí (-8\%), Cañar (-11\%), Pastaza (-14\%), Pichincha (-15\%). En esta última es donde queda la capital Quito. No obstante, hay regiones donde las mujeres trabajadores que tienen hijos ganan significativamente más que el resto de las mujeres. Este es el caso de Orellana, donde las madres ganan casi un $55 \%$ más que las no madres; le siguen Zamora (44\%) y Carchi (19\%). Por otro lado, en la provincia de Guayas, donde queda la ciudad más industrializada del Ecuador: Guayaquil, se calcula que las mujeres que tienen hijos ganan casi lo mismo que las no madres. Situación similar a la presentada en la región de Imbabura donde el diferencial salarial es de solo $0,12 \%$ a favor de las madres.

Estas diferencias regionales pueden estar determinadas por los niveles productividad o de especialización económica, lastimosamente este tema no ha sido investigado por la literatura reciente (Olarte y Peña, 2010). 


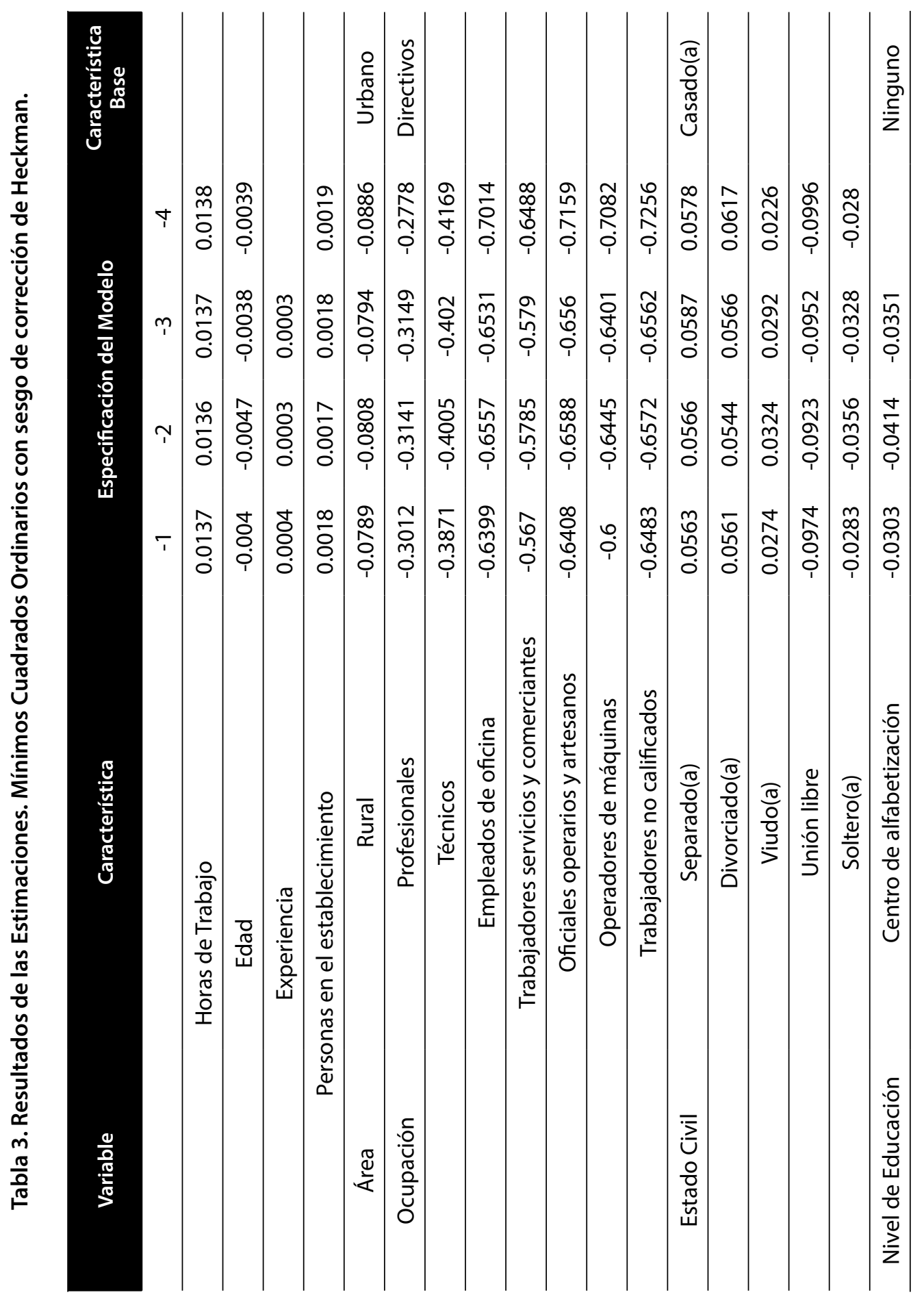




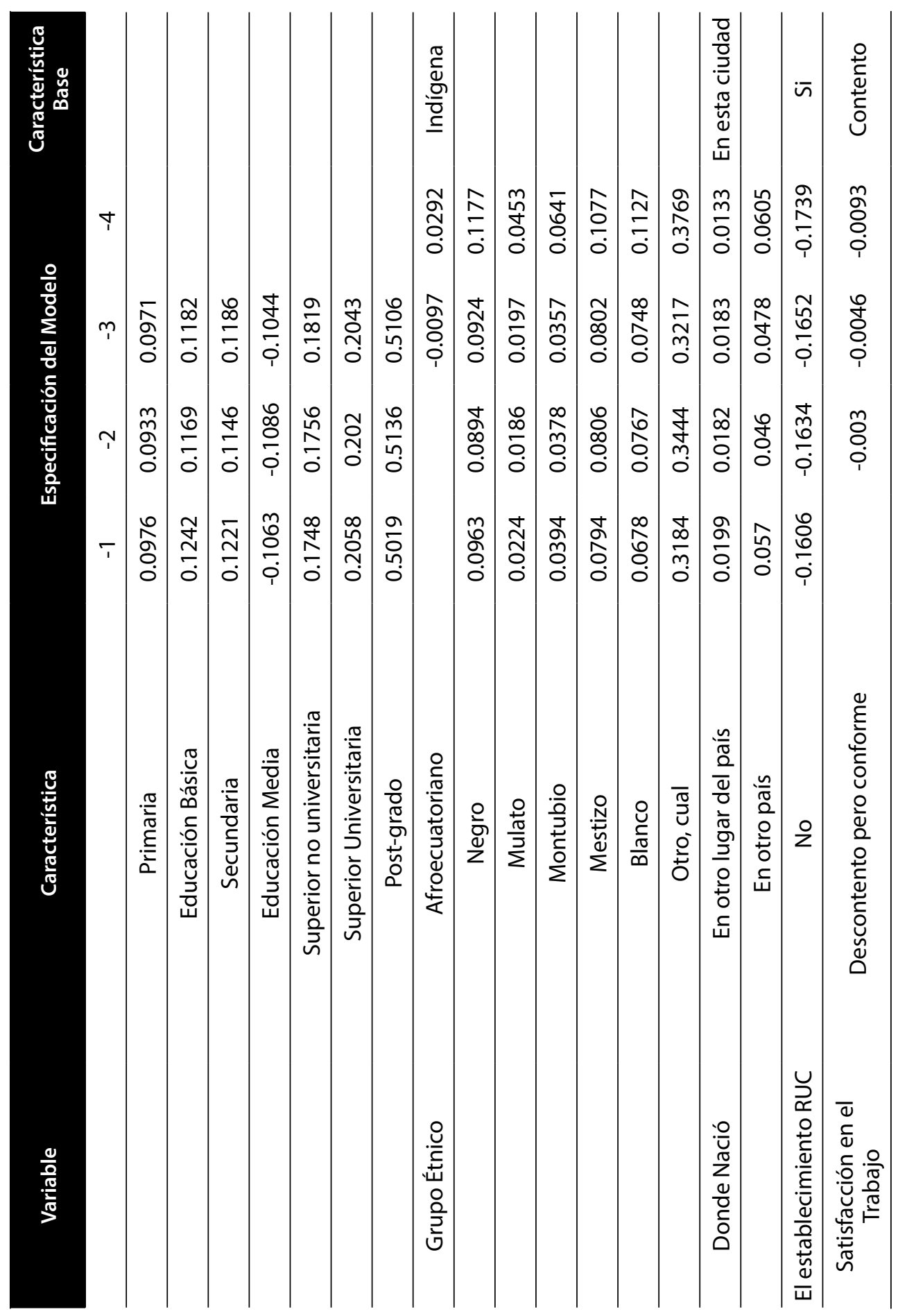




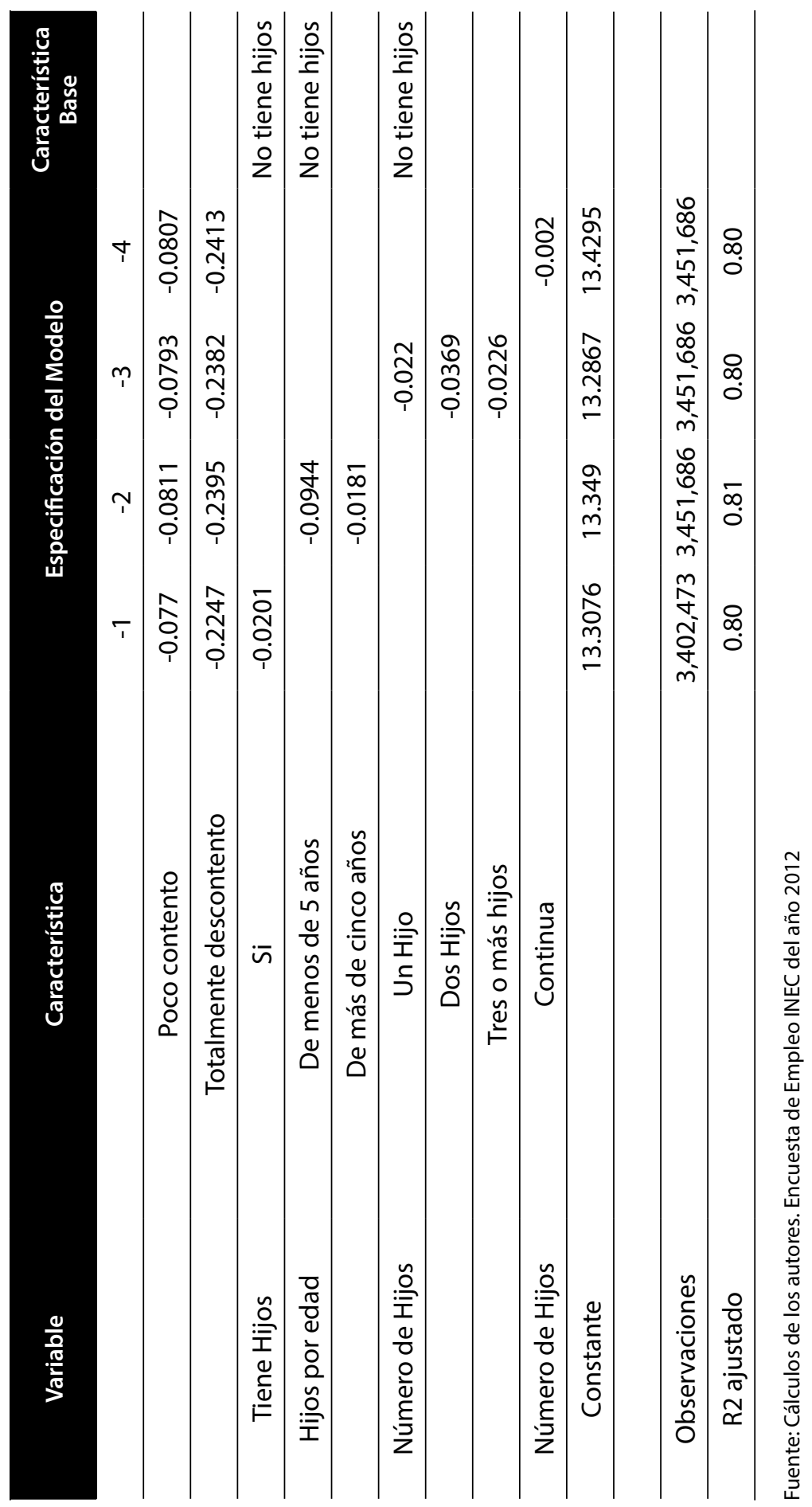


Cuando se revisan los otros tipos de especificaciones se pueden lograr análisis interesantes. Por ejemplo, cuando se tienen hijos mayores de cinco años (especificación 2), el salario de las madres corresponde a un $2 \%$ menos, mientras que las mujeres que tienen hijos de menos de cinco años, presentan una brecha de $10 \%$. Esto es debido a que niños más pequeños requieren una mayor atención de la madre y por ende mayores horas de tiempo en el hogar y menos en el trabajo, lo que las impulsa a buscar trabajos con horarios flexibles, con baja productividad e ingreso laboral.

Gráfica 1. Diferencial salarial por número de hijos

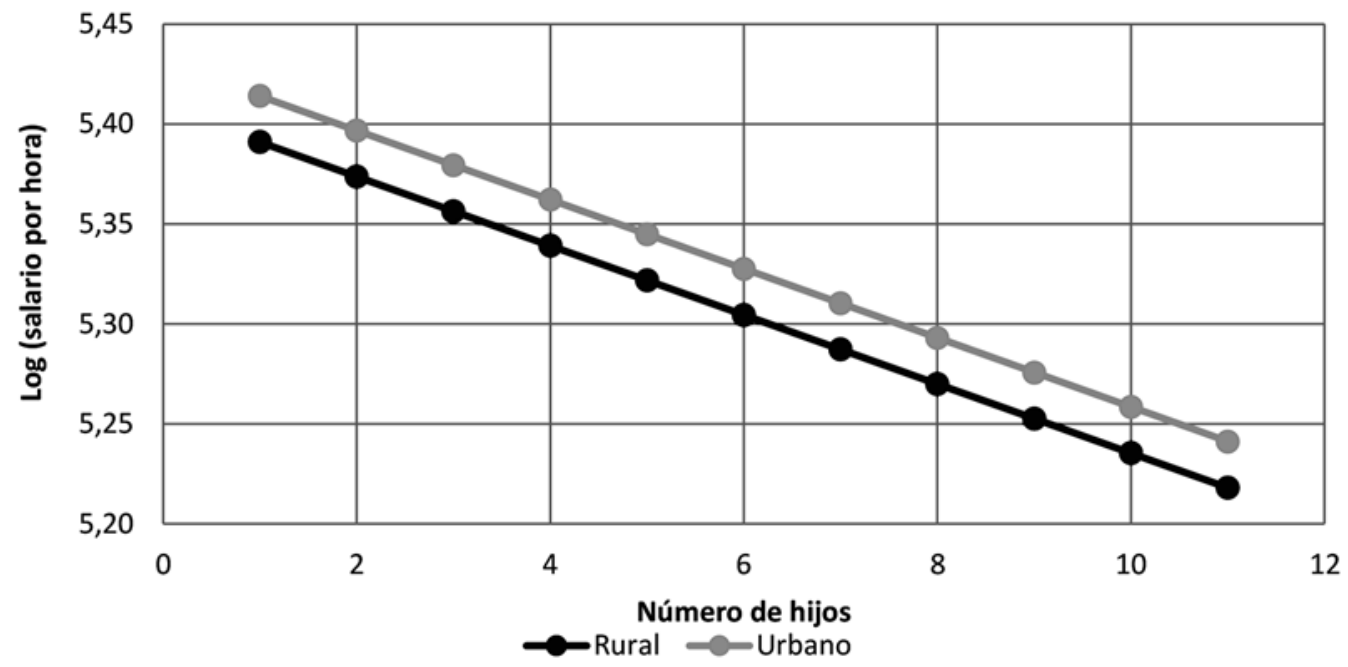

Fuente: Cálculos de los autores. Encuesta de Empleo INEC del año 2012

La especificación 3 permite estimar que si la mujer posee un hijo, el salario promedio se reduce en un 2,2\% en comparación con las mujeres que no tienen hijos. Si la mujer posee dos hijos la disminución es del 3,7\% y si tiene más de dos hijos la rebaja es del 2,26\%. Estos resultados colocarían al Ecuador dentro del grupo de países como Canadá, Finlandia y Suecia, para los cuales la penalización salarial correspondiente a dos hijos se encuentra en valores inferiores al 5\% y lejos del grupo del Reino Unido, Australia, Alemania y Estados Unidos para los cuales la brecha salarial es de $25.5 \%, 12 \%, 10.7 \%$ y $10.5 \%$, respectivamente (Olarte y Peña, 2010)

Por último, la especificación 4 permite calcular que por cada niño adicional, el salario de la mujer se reduce en un $0.002 \%$ en promedio. En la gráfica 1 se muestra el resultado de un ejercicio que consistió en interactuar el promedio del salario con la tenencia de hijos por el área donde vivía la mujer. En este sentido, se muestra que la caída del salario a medida que se tienen más hijos es constante, tanto a nivel urbano como rural. La brecha observada entre los salarios en el mercado rural y urbano es característico de los sistemas de producción duopolios en los diferentes 
países de Latinoamérica (Reyes, 2011). En Ecuador para 2012, el diferencial era cercano al 8\% según la encuesta empleada en este estudio.

Otros resultados de interés que se pueden extraer de las estimaciones son:

- La educación es la mejor inversión que pueden hacer las mujeres para incrementar su salario, esquemáticamente los resultados se muestran en la gráfica 2 . En este orden de ideas, la tenencia de un grado universitario incrementa en un $21 \%$ el salario en comparación con el de una mujer que no cuenta con ningún nivel educativo, si poseía un grado técnico es de un 17\%, si secundaria un $25 \%$ y si primaria un 9,7\%. Este efecto es un hecho ampliamente documentado en la literatura sobre determinantes de los ingresos laborales. (Psacharopoulos, 2004; Cohn y Addison, 1998; Asplund y Pereira, 1999, Harmon et al, 2001, 2003).

\section{Gráfica 2. Ganancias del capital humano en la población femenina trabajadora en Ecuador por nivel educativo.}

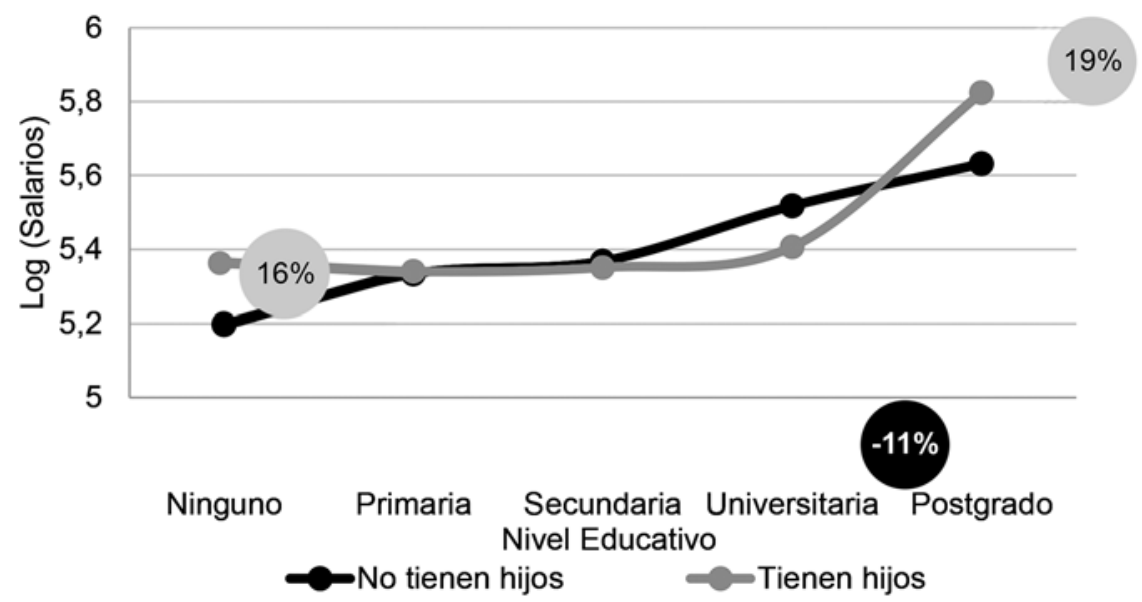

Fuente: Cálculos de los autores. Encuesta de Empleo INEC del año 2012

- Las madres solteras llegan a ganar entre un 2\% y un 3\% menos que las mujeres que trabajan y están con su pareja en el hogar. Esta tendencia se mantiene con otros tipos de estados civiles. Este resultado es similar al obtenido por Ugidos y de la Rica (1995).

- El grado de formalidad que tenga la empresa impacta de manera significativa el salario de las mujeres (Freije, 2001; Portes y Haller, 2004). Es así, como las mujeres que trabajan en una empresa con número de identificación de actividad económica (RUC) ganan un $16 \%$ por encima que las mujeres que laboran en establecimientos informales. Asimismo, el ocupar cargos en grandes empresas brinda la posibilidad de mejores ingresos laborales. En promedio por cada trabajador adicional que tienen la empresa donde laboran las mujeres, el salario se incrementa en un $0,018 \%$. 
Gráfica 3. Distribución geográfica de la brecha por maternidad en el Ecuador

\begin{tabular}{|l|c|}
\hline \multicolumn{1}{|c|}{ PROVINCIA } & $\%$ \\
\hline Azuay & $4.44 \%$ \\
\hline Bolívar & $11.41 \%$ \\
\hline Cañar & $-10.79 \%$ \\
\hline Carchi & $19.15 \%$ \\
\hline Chimborazo & $11.72 \%$ \\
\hline Cotopaxi & $13.74 \%$ \\
\hline El Oro & $-2.66 \%$ \\
\hline Esmeraldas & $-2.36 \%$ \\
\hline Guayas & $0.83 \%$ \\
\hline Imbabura & $0.12 \%$ \\
\hline Loja & $1.51 \%$ \\
\hline Los Ríos & $-1.52 \%$ \\
\hline Manabí & $-8.35 \%$ \\
\hline Morona Santiago & $-0.17 \%$ \\
\hline Orellana & $55.05 \%$ \\
\hline Pastaza & $-14.26 \%$ \\
\hline Pichincha & $-14.88 \%$ \\
\hline Sucumbíos & $18.60 \%$ \\
\hline Tungurahua & $19.48 \%$ \\
\hline Zamora Chinchipe & $44.37 \%$ \\
\hline
\end{tabular}

Fuente: Cálculos de los autores. Encuesta de Empleo INEC del año 2012

- Los sectores servicios (comercio, hoteles, restaurantes, transporte, financieros etc.) ofrecen a las mujeres los mejores ingresos laborales, con salarios entre $15 \%$ y $20 \%$ por encima del sector agrícola. García (1991), explica que estas brechas sectoriales se pueden deber al stock de capital implementado en los diferentes procesos de producción y a la cantidad de innovación y desarrollo que se haya realizado en estos sectores.

- En relación a la satisfacción laboral, las mujeres que dicen estar contentas con su trabajo ganan un $22 \%$ más de las que dicen estar totalmente descontentas con su trabajo. A pesar de este resultado, una parte de la literatura asociada al tema sobre los determinantes de la satisfacción laboral (Bañuelos al, 2005; Bòria-Reverter et al., 2012) parece indicar que la causalidad del efecto puede ser desde el salario hacia la satisfacción y no al contrario. 


\subsection{Descomposición de Oaxaca Blinder}

Encontradas las brechas salariales entre las madres y las no madres es necesario separar el efecto que es producido por la condición de maternidad y cual parte es producido por las variables no observables dentro del modelo.

En este orden de ideas, la tabla 4 muestra los resultados de la descomposición salarial a través de la metodología Oaxaca Blinder. Primero se obtienen los valores esperados de la variable dependiente (el logaritmo del salario) para cada grupo con las variables de control escogidas. Luego se estima la diferencia entre grupos. A continuación, se separan los efectos provenientes de los coeficientes y de la variable de control. Todos estos procesos se realizan por modelos, uno que no realiza la corrección del sesgo de selección y otro que sí lo corrige, tal y como se expuso en la parte metodológica.

Tabla 4. Resultados de la descomposición Oaxaca Blinder. Variable Dependiente: Logaritmo del Salario de las Mujeres trabajadoras por tenencia de Hijos.

\begin{tabular}{ccc}
\hline Grupo de Predicción & Sin corrección & Corregido Sesgo Selección \\
\hline Tienen Hijos & $5.352^{* *}$ & $5.334^{* *}$ \\
\hline No tienen Hijos & $5.361^{* *}$ & $5.259^{* *}$ \\
\hline Diferencia & $-1.11 \%^{* *}$ & $-7.56 \%^{* *}$ \\
\hline Dotación (Explicada) & $3.13 \%^{* *}$ & $-7.28 \%^{* *}$ \\
\hline Maternidad (No explicada) & $-4.24 \%^{* *}$ & $0.28 \%$ \\
\hline & ${ }^{* *}$ Es significativo al 1\%. \\
Fuente: Cálculos de los autores. Encuesta de Empleo INEC del año 2012
\end{tabular}

Se observa que sin la corrección la brecha por maternidad es del 1.11\%. Las características de las madres explican cerca de dos terceras partes de diferencial, con el 3,13\%, mientras que las variables no observables como la maternidad generan un diferencial negativo del $4,24 \%$.

Con la corrección del sesgo de selección, los resultados cambian drásticamente. Siendo el diferencial entre los valores esperados de los salarios del 7,56\% en contra de las mujeres que tienen hijos. Las características de las mujeres incluidas dentro del modelo son las causantes de la mayor parte de la brecha con el 7,28\% mientras que la maternidad solo es responsable del $0,28 \%$, no obstante este factor no resulto ser significativo desde el punto de vista estadístico. Este resultado es contrario al alcanzado con datos de 1999 por de Piras y Ripani (2005).

Por regiones (tabla 5), los análisis indican que 4 regiones tuvieron diferenciales salariales significativos entre madres y no madres: Pichincha (-36.58\%), Esmeraldas (-28.98\%), El Oro (-17.99\%), Guayas (-17.24\%). En todos los casos, a excepción de 
Esmeraldas, la brecha es explicada en su totalidad de manera significativa por la dotación de las mujeres que no tienen hijos, tales como la educación, la experiencia o las características de las empresas donde laboran. En el caso de la Provincia de Esmeraldas el diferencial es del 29\% en contra de las mujeres y está causada por su condición de ser madres u otras variables observables.

Tabla 5. Diferenciales de Salarios a partir de la descomposición Oaxaca Blinder con corrección de Heckman por provincia en el Ecuador.

\begin{tabular}{ccccccc}
\hline Provincia & Diferencial & Sig. & Dotación & Sig. & Maternidad & Sig. \\
\hline Azuay & 0.086 & 0.27 & 0.070 & 0.27 & 0.017 & 0.82 \\
\hline Bolívar & 0.255 & 0.05 & 0.065 & 0.66 & 0.190 & 0.25 \\
\hline Cañar & 0.218 & 0.09 & 0.173 & 0.12 & 0.045 & 0.72 \\
\hline Carchi & 0.044 & 0.76 & -0.033 & 0.81 & 0.077 & 0.52 \\
\hline Chimborazo & -0.156 & 0.30 & -0.036 & 0.68 & -0.120 & 0.43 \\
\hline Cotopaxi & 0.085 & 0.52 & 0.042 & 0.70 & 0.042 & 0.73 \\
\hline El Oro & -0.180 & 0.01 & -0.134 & 0.02 & -0.045 & 0.46 \\
\hline Esmeraldas & -0.290 & 0.05 & 0.006 & 0.95 & -0.296 & 0.03 \\
\hline Guayas & -0.172 & 0.01 & -0.163 & 0.00 & -0.009 & 0.85 \\
\hline Imbabura & -0.107 & 0.46 & -0.250 & 0.06 & 0.143 & 0.18 \\
\hline Loja & 0.002 & 0.99 & -0.148 & 0.15 & 0.151 & 0.16 \\
\hline Los Ríos & 0.095 & 0.36 & -0.006 & 0.93 & 0.101 & 0.32 \\
\hline Manabí & -0.191 & 0.14 & -0.124 & 0.09 & -0.068 & 0.60 \\
\hline Morona San & -0.108 & 0.70 & 0.564 & 0.18 & -0.671 & 0.14 \\
\hline Pastaza & 0.184 & 0.68 & -0.632 & 0.04 & 0.817 & 0.11 \\
\hline Pichincha & -0.366 & 0.00 & -0.410 & 0.00 & 0.045 & 0.50 \\
\hline Sucumbíos & -0.040 & 0.96 & 0.085 & 0.75 & -0.125 & 0.86 \\
\hline Tungurahua & 0.232 & 0.00 & 0.116 & 0.06 & 0.117 & 0.07 \\
\hline Zamora & -0.091 & 0.91 & 0.062 & 0.94 & -0.153 & 0.88 \\
\hline & & & & & 2012 & \\
\hline & & & & \\
\hline
\end{tabular}

Fuente: Cálculos de los autores. Encuesta de Empleo INEC del año 2012

Si la significancia está por debajo del 5\% (0.05) se considera que el resultado no es relevante desde el punto de vista estadístico. 


\section{Conclusiones}

En el presente estudio se analiza el efecto de la maternidad sobre los salarios en Ecuador en el 2012, estudiando sobre la tenencia del número de hijos y de la estructura de las edades de los mismos con base en la Encuesta de hogares realizadas por el INEC.

Las estimaciones con la metodología Oaxaca Blinder con corrección del sesgo de selección de Heckman sugieren que las mujeres que tienen hijos ganan en promedio 7,56\% menos que las no-madres. Luego de realizar otras especificaciones y controlar la edad de los hijos, se aprecia que las madres de niños de menos de 5 años ganan un $10 \%$ menos que las mujeres que no tienen hijos, mientras que las madres que tienen niños de más de 5 años, su salario disminuye en solo el $2 \%$. Por número, si la mujer tiene a su cuidado dos hijos la brecha salarial por maternidad promedio es del 3,7\% y si tiene más de dos hijos la rebaja salarial es del 2,26\%.

Los resultados muestran que la brecha salarial no se distribuye equitativamente para todas las provincias del Ecuador siendo las más afectadas por este fenómeno las localizadas en el Pichincha, Esmeraldas, Oro, Guayas con una baja del 14\% en el salario de las mujeres que tienen hijos frente a las que no tienen. Este diferencial está causado en su totalidad por las diferencias en las características de las mujeres ocupadas.

Con base en lo anterior, se afirma y de acuerdo a la literatura investigada que Ecuador es uno de los países donde la brecha por maternidad es más baja. Esto confirmaría que la diferencia de salarios entre hombres y mujeres en el Ecuador la cual es cercana al 10.1\% (Rivera, 2013) no está causada por el hecho implícito de que las mujeres tengan que dedicar más tiempo a sus hijos en el hogar, sino por la elección propia de los empleadores dado que observan las características socioeconómicas de las aspirantes.

En este sentido, si bien las políticas existentes como las licencias de maternidad remuneradas y la prohibición del despido a las embarazadas evitan las interrupciones de carrera y el flujo de ingresos en el corto plazo, las políticas orientadas a la disminución de la brecha por maternidad deben lograr que las madres tengan menores pérdidas en términos de experiencia laboral y educación en un horizonte más largo del tiempo especialmente para mujeres con hijos en edades inferiores a los cinco años.

Finalmente el presente artículo presenta limitaciones. La exploración de diferentes regiones presenta un problema de contextualización. Igualmente, el modelo no toma en cuenta el cambio en las características de los individuos en el tiempo. Es necesario la utilización de datos longitudinales que permitan el seguimiento de esta dinámica. 


\section{Referencias}

1. ASPLUND, Rita, y Pedro PEREIRA (1999). Returns to Human Capital in Europe. A Literature Review, Helsinki, ETLA.

2. ATUCHA, Ana (2009). Brechas salariales: discriminación o diferencias de productividad. Revista Momento Económico, (126).

3. BAÑUELOS, Alberto, DESCALS, Prada, CANTISANO, Gabriela y DOMÍNGUEZ, José (2005). Determinantes específicos de la satisfacción laboral, el burnout y sus consecuencias para la salud: un estudio exploratorio con funcionarios de prisiones. International Journal of Psychology and Psychological Therapy, 5(1), 71-81.

4. BAUM, Charles (2003). The effect of state maternity leave legislation and the 1993 Family and Medical Leave Act on employment and wages. Labour Economics, 10(5), 573-596.

5. BAXTER, Janeen (1992). Domestic Labour and Income Inequality. Employment and Society, 6, 229-249.

6. BECKER, Gary (1985). Human capital effort, and the sexual division of labour. Journal of Labour Economics, 3, 33-58.

7. BÒRIA-REVERTER, Sefa; CRESPI-VALLBONA, Montserrat y MASCARILLA-MIRÓ, Oscar (2012). Variables determinantes de la satisfacción laboral en España. Cuadernos de economía, 35(97), 9-16.

8. BUDIG, Michelle J., y Paula ENGLAND (2001). The Wage Penalty for Motherhood. American Sociological Review, 66, (2), 204-225.

9. COHN, Elchanan, y John T. ADDISON (1998). The Economics Returns to Lifelong Learning in OECD Countries, Education Economics, 6, pp. 253-307.

10. DAYMONT, Thomas y Paul J. ANDRISANI (1984). Job preferences, college major, and the gender gap in earnings. Journal of Human Resources, 408-428.

11. ESPINOZA, Nereyda (2009) Estimación de la Brecha Salarial entre Hombres y Mujeres: Un análisis por Cuantiles para el Ecuador. Económicas. Escuela Superior Politécnica del Litoral (ESPOL), 2-20.

12. ESQUIVEL, Valeria (2007). Género y diferenciales de salarios en la Argentina. En M. Novick y H. Palomino, edit. Estructura productiva y empleo: un enfoque transversal. Buenos Aires, Argentina: Ministerio de Trabajo, Empleo y Seguridad Social.

13. FREIJE, Samuel (2001). El empleo informal en América Latina y el Caribe: causas, consecuencias y recomendaciones de política. Banco Interamericano de Desarrollo, Primer Seminario

14. FUENTES, Jeanette, Amalia PALMA y Rodrigo MONTERO (2005). Discriminación salarial por género en Chile: una mirada global. Estudios de Economía, 32(2).

15. GARCÍA, Jaume (1991). Una interpretación de las diferencias salariales entre sectores. Investigaciones Económicas (Segunda época), 15(1), 143-167.

16. HARMON Colm, lan WALKER, y Niels C. WESTERGÅRD-NIELSEN (2001). Education and earnings in Europe. A cross country analysis of the return to education. Cheltenham: Edward Elgar.

17. HARMON, Colm, Hessel OOSTERBEEK, y lan WALKER (2003). The returns to education: microeconomics. Journal of Economic Surveys, 17, pp. 115-155. 
18. HARKNESS, Susan, y Jane WALDFOGEL (2003). The family gap in pay: evidence from seven industrialized countries. Research in Labor Economics, 22, 369-414.

19. HECKMAN, James. (1979). Sample selection bias as a specification error. Econometrica, 47, 153-161

20. JACOBSEN, Joyce P., y Laurence M. LEVIN (1995). Effects of intermittent labor force attachment on women's earnings. Monthly Labor Review, 118, 14-19.

21. JOSHI, Heather, and Marie-Louise NEWELL. (1989). Pay Differentials and Parenthood: Analysis of Men and Women Born in 1946. University of Warwick Institute for Employment Research.

22. KORENMAN, Sanders, y David NEUMARK (1992). Marriage, motherhood, and wages. Journal of Human Resources, 27, 233-255.

23. LAI, Yu-Cheng, y Stanley MASTERS (2005). The effects of mandatory maternity and pregnancy benefits on women's wages and employment in Taiwan, 1984-1996..Industrial and Labor Relations Review, 274-281.

24. MARTÍNEZ Jasso y Flores ACEVEDO (2004). La brecha salarial en México con enfoque de género: capital humano, discriminación y selección muestral. Ciencia Uanl, 7(1).

25. MINCER, Jacob. (1974). Schooling, Experience and Earnings. National Bureau of Economic Research.

26. MOLINA, José Alberto, y Víctor M. MONTUENGA (2008). The Motherhood Wage Penality in a Mediterranean Country: The Case of Spain. IZA Discussion Papers 3574, Institute for the Study of Labor, Bonn (Germany).

27. MONTENEGRO, Claudio (2001) Wage Distribution in Chile: Does Gender Matter? A Quantile Regression Approach. Banco Mundial, 3-35.

28. OLARTE, Liliana y PEÑA, Ximena. (2010). The Effect of Motherhood on Wages in Colombia. Documento CEDE. Universidad los Andes.

29. PEÑAS, Ignacio (2002). La discriminación salarial por razones de género: un análisis empírico del sector privado en España. Reis, 171-196.

30. PIRAS Claudia y Laura RIPANI, (2005). The Effects of Motherhood on Wages and Labor Force Participation: Evidence from Bolivia, Brazil, Ecuador and Peru. IDB Publications 49638, Inter-American Development Bank.

31. PSACHAROPOULOS, George; Harry Anthony PATRINOS (2004). Returns to investment in education: $a$ further update. Education Economics, 12(2), 111-134.

32. PORTES, Alejandro, y HALLER, William. (2004). La economía informal. CEPAL

33. REYES Giovanni (2011). Centros urbanos de América latina 1997, 2006: disparidades salariales según género y crecimiento económico. Revista de Ciencias Sociales, (131-132).

34. RIVERA, Jairo (2013). Teoría y Práctica de la Discriminación en el Mercado Laboral Ecuatoriano (20072012). Analítika, 5(1), 3-18.

35. TODD, Erin (2001). Educational attainment and family gaps in women's wages: evidence from five industrialized countries. Luxembourg Income Study, LIS WP. N. 246, Luxembourg.

36. UGIDOS, Arantza y DE LA RICA, Sara (1995). Son las diferencias en capital humano determinantes de las diferencias salariales observadas entre hombres y mujeres. Investigaciones económicas, 19(3), 395-414.

37. WALDFOGEL, Jane. (1995). The price of motherhood: family status and women's pay in a young British cohor. Oxford Economic Papers, 47, (4), 584-610.

38.

(1997). Working mothers then and now: a cross-cohort analysis of the effects of maternity leave on womens pay. [Unpublished] 1996. Presented at the Annual Meeting of the Population Association of America New Orleans Louisiana, May 9-11 1996. 